\title{
RR-APET - Heart rate variability analysis software
}

\author{
Meghan McConnell ${ }^{\mathrm{a}, *}$, Belinda Schwerin ${ }^{\mathrm{a}}$, Stephen So $^{\mathrm{a}}$, Brent Richards $^{\mathrm{b}}$ \\ ${ }^{a}$ Signal Processing Laboratory, Griffith School of Engineering and Built Environment, Griffith University, Southport QLD 4222, Australia \\ ${ }^{b}$ Gold Coast University Hospital, Intensive Care, Southport QLD 4215, Australia
}

\begin{abstract}
Background and Objectives: Heart rate variability (HRV) has increasingly been linked to medical phenomena and several HRV metrics have been found to be good indicators of patient health. This has enabled generalised treatment plans to be developed in order to respond to subtle personal differences that are reflected in HRV metrics. There are several established HRV analysis platforms and methods available within the literature; some of which provide command line operation across databases but do not offer extensive graphical user interface (GUI) and editing functionality, while others offer extensive ECG editing but are not feasible over large datasets without considerable manual effort. The aim of this work is to provide a comprehensive open-source package, in a well known and multi-platform language, that offers considerable graphical signal editing features, flexibility within the algorithms used for R-peak detection and HRV quantification, and includes graphical functionality for batch processing. Thereby, providing a platform suited to either physician or researcher.
\end{abstract}

Methods: RR-APET's software was developed in the Python language and is modular in format, providing a range of different modules for established R-peak detection algorithms, as well as an embedded template for alternate algorithms. These modules also include several easily adjustable features, allowing the user to optimise any of the algorithms for different ECG signals or databases. Additionally, the software's user-friendly GUI platform can be operated by both researchers or medical professionals to accomplish different tasks, such as: the in-depth visual analysis of a single ECG, or the analysis multiple signals in a single iteration using batch processing. RR-APET also supports several popular data formats, including text, HDF5, Matlab, and Waveform Database (WFDB) files.

Results: The RR-APET platform presents multiple metrics that quantify the heart rate variability features of an Rto-R interval series, including time-domain, frequency-domain, and nonlinear metrics. When known R-peak annotations are available, positive predictability, sensitivity, detection error rate, and accuracy measures are also provided to assess the validity of the implemented R-peak detection algorithm. RR-APET scored an overall usability rating of 4.16 out of a possible 5, when released on a trial basis for user evaluation.

Conclusions: With its unique ability to both create and operate on large databases, this software provides a strong platform from which to conduct further research in the field of HRV analytics and its correlation to patient healthcare outcomes. This software is available free of charge at https://gitlab.com/MegMcC/rr-apet-hrv-analysis-software and can be operated as an executable file within Windows, Mac and Linux systems.

Keywords: Heart rate variability (HRV), R-peak detection, Analysis software, Python 3, Computer program

\section{Introduction}

HRV is a common phrase used to characterise fluctuations that occur within heart rate (HR) [1]. There are a variety of popular HRV metrics used to quantify the temporal variation between successive heartbeats. This time sequence can be referred to as both the interbeat interval (IBI) series, or the R-to-R interval (RRI) series. These metrics, which can be measured in various domains, are most often implemented to assess autonomic modulation in a non-invasive manner [2].

\footnotetext{
* Corresponding author

Email address: m.mcconnell@griffith.edu.au (Meghan McConnell)
}

HRV was recognised as an important biomarker after its initial use in the 1980 s, where it was confirmed as an independent and strong predictor for determining the risk factor of sudden cardiac death following acute myocardial infarction $[2,3,4]$. Since then, HRV has been found to be useful in a variety of clinical scenarios, such as: mortality prediction for patients presenting with sepsis [5], prognostic capacity following acute myocardial infarction [6], indicator of concussion and post-concussion rehabilitation [7], and as a trans-diagnostic biomarker of psychopathology [8]. Other non-clinical applications have also been linked to HRV such as: sports physiology [9], fatigue, stress, sleep, age, and gender [10]. 
However, HRV is not a single metric that can be recorded directly from a patient; it instead must be extracted as a combination of secondary metrics. HRV can be quantified by analysing a series of sequential IBIs. Many devices and acquisition systems are capable of generating accurate RRI series by implementing specialty beat detection methods on measured bio-signals such as an electrocardiogram (ECG) or a photoplethysmogram (PPG). With the increased popularity of HRV metrics, there has come a rise in analytical software tools tailored towards the extraction of these metrics, in both commercial and noncommercial domains. Some commercially available HRV software tools include: Kubios HRV Premium ${ }^{1}[11,12]$, LabVIEW [13], Plux [14], and CODESNA [15]. Whilst these packages are highly sophisticated, offering GUI based functionality, and time-domain, frequency-domain and nonlinear analyses, they are also restrictive in their use due to expensive licensing requirements. However, several non-commercial HRV packages are also available.

A majority of the non-commercial software packages have been developed in the Matlab programming environment [16], such as: ECGLab [17], Kardia [18], ARTiiFACT [19], and HRVTool [20]. ECGLab supports a variety of ECG formats including PhysioNet, ISHNE and FDA XML HL7, and is able to calculate common timedomain metrics, and perform spectral analysis. It is open source, operated using a GUI, but does not support batch processing. Similarly, Kardia is also an open-source, GUI operated HRV analysis package. Whilst able to perform batch processing without significant manual input, the major drawback of Kardia is that it is only able to process IBI series that have been stored using Matlab's matfile format. ARTiiFACT is operated through it's well designed GUI, and offers IBI detection, extensive artifact processing, and HRV quantification in both the frequency and time domain; however, does not support batch processing or PhysioNet's wfdb file format. HRVTool was developed as an open source toolbox for the Matlab environment. It is able to be operated within a GUI and offers extensive ECG editing features, with HRV metrics calculable in the time and frequency domains. Whilst batch processing is possible using the toolbox, script implementation is required. Another major drawback of software packages developed within the commercialised Matlab software is that a paid license is required for the use of this programming environment.

HRV analysis packages developed in other programming environments include: HRV toolkit developed by the PhysioNet Project [21], rHRV [22], and gHRV [23]. HRV toolkit is an extensive open source HRV analysis software package developed in the C-language, that can be used to visual the IBI time series, remove outliers, and calculate both time and frequency domain metrics. The major disadvantage of this package is that it is run using command-line

\footnotetext{
${ }^{1}$ Kubios HRV Standard is available without cost but does not contain all of the features available in Kubios HRV Premium.
}

prompts, within a terminal or shell-script environment, which restricts its use to researchers (or cardiologists) who are experienced programmers. rHRV is another extensive open-source toolbox developed in $\mathrm{R}$, composed of various powerful functions. However, like HRV toolkit, it is restrictive due to the required programming skills. Finally, gHRV is GUI-based program developed in the Python language, that allows the in-depth analysis of ECG signals, with time-domain, frequency-domain and nonlinear HRV quantification metrics available. Furthermore, gHRV includes significance analysis of its HRV metrics, however, it does not offer batch processing.

In this paper, we introduce the $R R-A P E T$ HRV analysis software which is a multi-platform, easy to use software tool that incorporates both R-peak detection modules and various HRV analysis metrics, including time-domain, frequency-domain and nonlinear techniques. $R R-A P E T$ is developed as an open source package, key with the objective of stimulating further work in this important research area. One of its key features is that the entire project has been developed as a stand-alone, packaged, executable program, written in the Python 3 language ${ }^{2}$, allowing all required software to be downloaded without the need for any paid subscription. By providing both the source code and an executable version of RRAPET, we have catered to a range of skill levels in potential users. Inexperienced users are able to operate all of RR-APET's features within the GUI platform including batch processing, whilst experienced users are provided with the opportunity to adapt and upgrade the base program in order to suit their specific needs without relying on updates. Furthermore, this software offers the choice of three R-peak detection techniques derived from well established methods [24, 25, 26] and a template to enable the implementation and testing of any other detection method of interest. It is the combination of all of these features being available in one analysis tool that gives $R R-A P E T$ a clear advantage over the software packages presently available.

\section{Computational methods}

In this section, we provide an overview of the computational methods which form the framework of $R R-$ $A P E T^{\prime} s$ software package. A representation of the major components of the package is shown in Fig. 1 . In general, the algorithm library contains scripts for R-peak detection, whilst the testing library contains scripts for the computation of analytical metrics. The HRV analysis parameters included in RR-APET are implemented using the guidelines given in $[2,5,27]$.

\footnotetext{
${ }^{2}$ All references to "Python" from this point onward imply that we are using Python 3.
} 


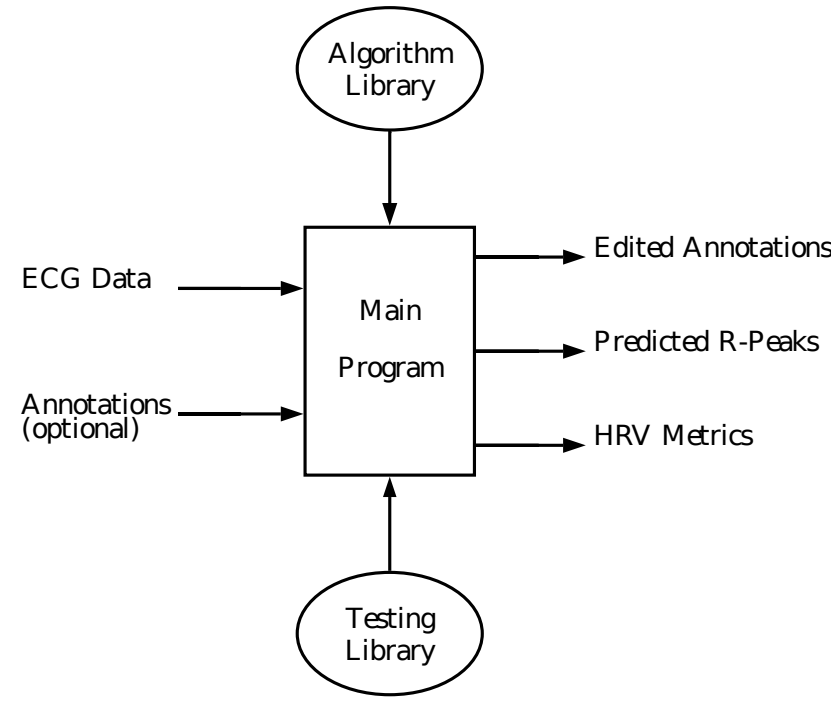

Fig. 1: Main components of the RR-APET software package.

\subsection{Algorithm Library}

The Algorithm library includes various Python based detection algorithm modules which can be used to process raw ECG signals and detect QRS complexes. Three detection algorithms are provided within the package. These algorithms were derived from established techniques, such as: the Pan Tompkins method [24], a method based on the implementation of K-means clustering [25] and a method based on the implementation of the Hilbert transform [26]. The reliability of each detection algorithm is detailed in Section 5.1. The design of these modules is highly flexible, and allows the user to define inputs for their own digital filters, variable thresholds, peak selection criteria, or to simply apply the preset values. These parameters can be accessed within the Program Preferences option of the GUI. Furthermore, there is a helpful template included for users to write and test their own R-peak detection algorithms.

\subsection{Testing Library}

The 'Testing library' contains the modules implemented by RR-APET to analyse the performance of R-peak detection algorithms with reference to true R-peak locations, as given by the annotation files. Consequently, this type of testing is viable only for data sets that are pre-annotated. This feature would be utilised to assess the validity of untested detection algorithms, such as a novel algorithm designed by the user. It also contains scripts to analyse RRI series using several key HRV metrics, including those calculated in both the timedomain, and the frequency-domain, as well as through the implementation of nonlinear methods. These tests are viable for both imported or predicted RRI series. The algorithms implemented here were validated by testing against established software, with results of these tests presented in Section 5.2.

\subsubsection{Detection algorithm analysis metrics}

Testing is performed using a beat-by-beat comparison method, where the predicted R-peaks are compared to the R-peak annotations, and categorised into three groups: True Positives (TP), False Positives (FP) and False Negatives $(\mathrm{FN})$.

For a detected R-peak prediction to be classified as a $\mathrm{TP}$, it must occur within a given precision window of the annotated R-peak for the corresponding heartbeat. To be classified as a FP, the R-peak prediction must occur in a position where no $\mathrm{R}$-wave is present. Finally, FNs are classified when an $\mathrm{R}$-wave is present in the signal but the algorithm fails to predict the corresponding R-peak. Fig. 2 visually represents the areas of prediction for each category.

The performance of the algorithms are then rated using the number of TP, FP, and FN classifications to produce four metrics: positive predictability $(\mathrm{P}+)$, sensitivity $(\mathrm{Se})$, accuracy (Acc), and detection error rate (DER). These metrics are calculated using the following equations:

$$
\begin{gathered}
P+=\frac{T P}{T P+F P} \times 100 \%, \\
S e=\frac{T P}{T P+F N} \times 100 \%, \\
A c c=\frac{T P}{T P+F P+F N} \times 100 \%, \\
D E R=\frac{F P+F N}{T P} \times 100 \% .
\end{gathered}
$$

The precision window is set to the default value of $0.15 \mathrm{~s}$ to match that found within the commonly implemented in WFDB beat-by-beat (bxb) comparator ${ }^{3}[21]$. This value can be reduced at the users discretion in order to access

\footnotetext{
${ }^{3}$ WFDB's bxb was developed in accordance to the standards set in ANSI/AAMI EC38:1998, the American National Standard for Ambulatory ECGs, and in ANSI/AAMI EC57:1998, the American National Standard for Testing and Reporting Performance Results of Cardiac Rhythm and ST Segment Measurement Algorithms.
}

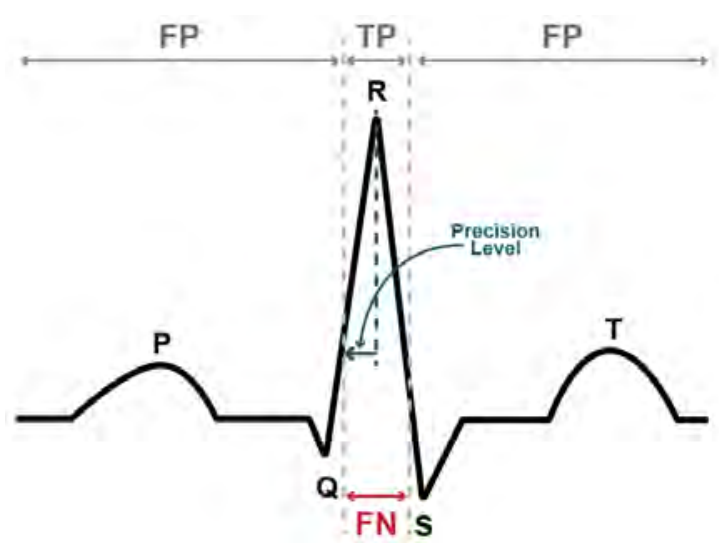

Fig. 2: Example of FP, FN and TP regions. 
the viability of $\mathrm{R}$-peak detection algorithms, rather than QRS detection algorithms.

\subsubsection{Time-domain methods}

When evaluating HRV within the time-domain, there are several key metrics that can be calculated, which give perspective on different types of variability. These calculations are conducted on the RRI series [2]. The simplest measure used to analyse variability in heart rate is the mean $\mathrm{RR}$ interval value $(\overline{\mathrm{RRI}})$. The standard deviation of the RRI series (SDNN) is an important measure as it reflects variability of cyclic components within the period of recording. This metric however, is dependent upon the length of the analysed recording [28], and so standardised recording lengths were devised to be five minutes for shortterm recordings and 24 hours for long-term recordings [2].

One metric that can be calculated from a long-term recording is the root mean square of successive $R R$ interval differences (RMSSD), a measure which provides an estimate of short-term HRV. Another metric used to evaluate HRV is pNN50, which is the ratio between the number pairs of successive RR intervals that differ by more than $50 \mathrm{~ms}$ (NN50) and the total number of RR intervals in the series (M). pNN50 is found as,

$$
\begin{aligned}
N N 50_{i}= & \left\{\begin{array}{ll}
1, & \text { if }\left|R R I_{i+1}-R R I_{i}\right| \geq 50 \times 10^{-3} \\
0, & \text { otherwise }
\end{array},\right. \\
& \text { for } \quad i=1, \ldots, M-1,
\end{aligned}
$$

and

$$
p N N 50=\frac{1}{M} \sum_{i=1}^{M-1} N N 50_{i},
$$

where $i$ is the $i$-th point in the RRI series.

The total monitoring period of a long-term recording can also be broken into segments to find other useful variables. Two of the most commonly derived variables include the SDNN index, which is calculated by taking the mean SDNN value of successive five minute intervals, and SDANN, which is the standard deviation of the average $\mathrm{RR}$ interval calculated over five minute intervals; both are calculated over a minimum of 24 hours. The SDNN index indicates the change in $\mathrm{HR}$ due to cycles shorter than five minutes, whilst SDANN value gives an estimate of variability due to cycles longer than five minutes [2].

\subsubsection{Frequency-domain methods}

Frequency domain techniques that are implemented to analyse HRV, usually stem from the analysis of the signal's power spectral density (PSD), which is a measure of how power is distributed as a function of frequency [2]. An estimate of PSD can be obtained through a variety of mathematical methods; this software offers a choice between both Welch's, Blackman-Tukey's, and LombScargle's periodograms, as well as from autoregressive (AR) modelling. However, a majority of these methods require a signal that is uniformly sampled, of which the RRI series is not. Interpolation of the RRI series allows it to be re-sampled evenly prior to spectral estimation. For the purposes of this software, the required interpolation was achieved by implementing cubic spline interpolation from the Scipy library of Python, with step size of 0.5 .

Welch's periodogram is produced by first sectioning the re-sampled series into $M$ segments of length $L$, that overlap by $O$ points. The fast Fourier transform (FFT) and desired windowing technique is applied to each segment in order to produce a modified periodogram, with the final PSD being generated by taking the average of the modified periodograms [29]. By default, RR-APET uses a window size of $L=1200$, and a window shift of $O=600$ when estimating the periodogram using Welch's method. Similarly, the Blackman-Tukey method applies a Fourier transform to estimate the PSD, but rather that applying it to the re-sampled series, the transform is applied to a windowed estimate of the auto-correlation function for a given number of lags, $K$ [30]. By default, RR-APET uses $K=10$ when estimating the periodogram using Blackman-Tukey's method. Both methods provide lower variance estimates of PSD when compared with the conventional periodogram method.

In autoregressive (AR) spectral estimation, an all-pole filter (with system function $H(z)$ ) is designed such that its output is the re-sampled series while the input signal is white noise. This is done by estimating the autocorrelation coefficients of the re-sampled series and then solving the Yule-Walker equations in order to obtain the filter coefficients of $\mathrm{H}(\mathrm{z})$ [31]. The PSD estimate is obtained as the squared magnitude response of the all-pole filter, i.e. $|H(z)|^{2}$. Being a parametric method, the PSD estimates that are obtained using AR techniques tend to be smoother and have better frequency resolution than the periodogram-based methods.

Conversely, the Lomb-Scargle periodogram method can be applied to signals with uneven, temporal sampling, and therefore interpolation of the RRI series is not required. It is done by using the Lomb-Scargle equations in order to estimate the normalised power, as a function of angular frequency, for all tested periods [32]. This method also accounts for the phase-shifts caused by unequally sampled data.

In order to quantitatively analyse the desired signal's PSD, it is first broken into three distinct frequency bands: very low frequency (VLF) from 0.003-0.04 Hz, low frequency (LF) from 0.04-0.15 Hz and high frequency (HF) from 0.15-0.4 Hz [2]. Physiologically, the LF band has been correlated to parasympathetic stimulation of the autonomic nervous system (ANS), whilst the HF band has been correlated to sympathetic stimulation of the $\mathrm{ANS}^{4}$ [10]. These frequency bands are then analysed to determine

\footnotetext{
${ }^{4}$ It has also been argued that the LF band correlates to both parasympathetic and sympathetic stimulation, and that the HF band correlates both sympathetic stimulation and breathing [33].
} 
the relative power, absolute power, and peak frequency within each band. These values can be used to assess the autonomic parasympathetic-sympathetic balance due to their physiological correlations.

\subsubsection{Nonlinear methods}

Nonlinear techniques for analysing HRV are often quite useful as they can help to characterise the more complex dynamics of the heartbeat that occur during cardiovascular regularisation, where linear methods can often be insufficient [34]. The first nonlinear technique included in $R R-A P E T^{\prime} s$ software is the recurrence plot (Fig. 3), as well as a quantified analysis of its graphical features. Recurrence plots offer a two-dimensional visualisation of the trajectory's periodic nature in an $m$ dimensional phase space. It does this by depicting sets of recurrence pairs, i.e. sets of times at which the trajectory is considered to have returned to the same space [27]. In order to represent a scalar time series $x(i)$ as a multi-dimensional process, a trajectory vector $\mathcal{X}_{i}(k)$ is implemented using,

$$
\mathcal{X}_{i}(k)=x(i+k * L), \quad \text { for } \quad k=0, \ldots, m-1,
$$

where $m$ is the embedding dimension, $L$ is the lag, $i$ is the time series index, and $k$ is the sample index within the embedded vector [27]. Every trajectory point, $\mathcal{X}_{i}$, is then compared to all other trajectory points, $\mathcal{X}_{j}$, to determine the distance, $\operatorname{dist}\left(\mathcal{X}_{i}, \mathcal{X}_{j}\right)$, between each vector using,

$$
\operatorname{dist}\left(\mathcal{X}_{i}, \mathcal{X}_{j}\right)=\sqrt{\sum_{k=1}^{m-1} \mathcal{X}_{i}(k)-\mathcal{X}_{j}(k)}
$$

Any two points $\left(\mathcal{X}_{i}, \mathcal{X}_{j}\right)$ that occur within a fixed decision boundary radius, $r$, of each other are considered recurrent

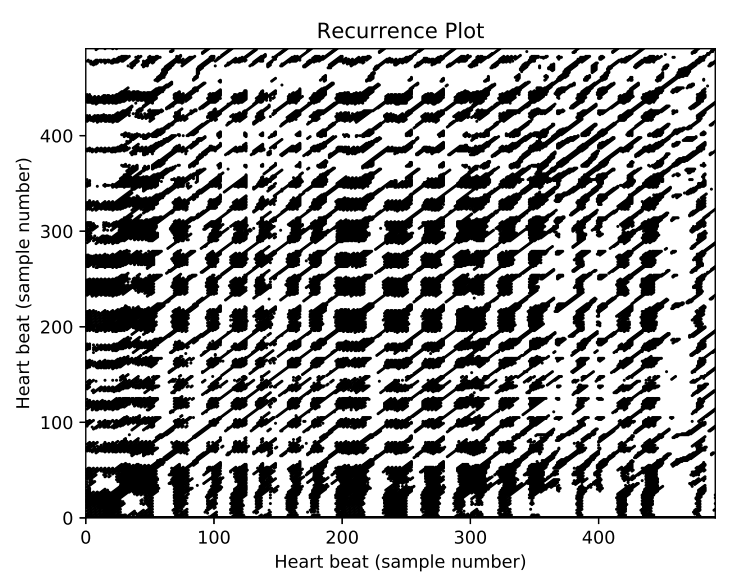

Fig. 3: An example of a recurrence plot generated using a standard RR interval series. (i.e. $R P(i, j)=1)$ [27]. For an $N$ point long time series,

$$
\begin{aligned}
R P(i, j)= \begin{cases}1, & \text { ifdist }\left(\mathcal{X}_{i}, \mathcal{X}_{j}\right) \leq r \\
0, & \text { otherwise }\end{cases} \\
\text { for } \quad i=0,1,2, \ldots, N-m-1, \\
\quad j=0,1,2, \ldots, N-m-1,
\end{aligned}
$$

where $R P(i, j)$ is the recurrence plot matrix.

In deterministic signals, the recurrence plot will show diagonal lines parallel to the main diagonal, which correspond to areas where sequences which are repeated; in contrast, randomness is suggested when the plot does not incorporate this diagonal pattern [35]. These qualitative measures lead to the development of standard practice recurrence quantification analysis (RQA) metrics $[27,36$, 37]. In regards to HRV, a recurrence plot with many diagonals present suggests a patient with limited variation in HR; whereas, a recurrence plot with few diagonals present indicates a patient who has large variation in their HR. The following RQA metrics have been included in the provided software: the recurrence rate $(\% R E C)$, the determinism as percentage of recurrence points which form diagonal lines $(\% D E T)$, the mean length of structures both diagonal $\left(L_{\text {mean }}\right)$ and vertical $\left(V_{\text {mean }}\right)$, and the max length of structures both diagonal $\left(L_{\max }\right)$ and vertical $\left(V_{\max }\right)$.

The second nonlinear technique included in $R R-$ $A P E T^{\prime} s$ software is detrended fluctuation analysis (DFA). DFA can be applied to both stationary and non-stationary processes, in order to reveal correlations within the data over varying ranges [38]. In order to apply DFA to an RRI time series, the series is first integrated using,

$$
y(k)=\sum_{i=1}^{k}\left[R R I_{i}-R R I_{\text {mean }}\right],
$$

where $R R I_{i}$ is the $i$-th point of the RRI time series, $R R I_{\text {mean }}$ is the mean of all of the points within the time series [5]. The integrated series, $y(k)$, is then detrended by splitting the series into $n$ length boxes and applying,

$$
F(n)=\sqrt{\frac{1}{N} \sum_{k=1}^{N}\left[y(k)-y_{n}(k)\right]^{2}},
$$

where $y_{n}(k)$ is the local trend (calculated by fitting a linear regression model to each box), and $N$ is the length of the time series [5]. The final DFA is conducted by producing a log-log plot of the detrended series $F(n)$ versus box length $n$.

In regards to $\mathrm{HRV}$, if correlation is revealed by the DFA, it means that RRI's occurring in a given time interval are dependent on previous segments, to some degree [34]. Short-term fluctuations, denoted as $\alpha_{1}$, are considered to occur in the 4 to 15 beat range and correlate to both sympathetic and vagal modulation. Whilst long-term 
Poincare Plot

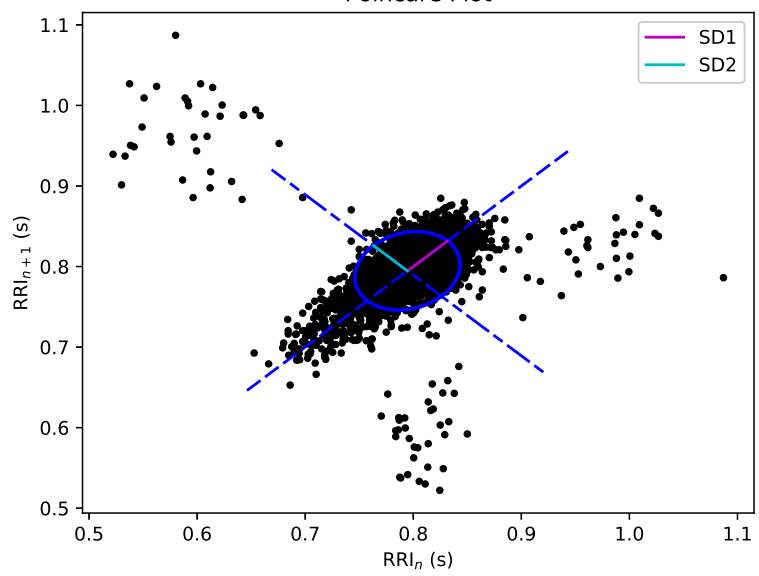

Fig. 4: A standard Poincaré plot of an RR interval series.

fluctuations, denoted as $\alpha_{2}$, are considered to occur in the 16 to 64 beat range and correlate to only sympathetic modulation [34, 39]. These ranges were selected to match those specified within the popular Kubios software [12]. In terms of the DFA plot, $\alpha_{1}$ and $\alpha_{2}$ are considered to be the gradients found when fitting a linear regression model to their respective ranges.

The third nonlinear technique included in $R R-A P E T^{\prime} s$ software is the Poincaré plot. This plot graphically represents the correlation between consecutive $\mathrm{RR}$ intervals [12]. To construct a Poincaré plot (Fig. 4), each RR interval point $\left(R R I_{i}\right)$ is plotted against the next point $\left(R R I_{i+1}\right)$ over the entire RR interval series [40]. In order to quantify the plot, an ellipse is fitted to the data points where the centre coincides with mean RR interval length for the ECG. The height and width of the ellipse are determined by taking the standard deviation along both the longitudinal axis $(S D 1)$ and the transverse axis $(S D 2)$ [41]. $S D 1$ reflects the short-term variability in the RRI series, whereas $S D 2$ reflects long-term variability [12].

\section{Software description}

In this work, a software package, $R R-A P E T$, has been developed. This software enables users to visually analyse, edit, or create RR-interval (RRI) series from raw ECG signals. Additionally, the user is able to analyse generated (or imported) RRI series using the methods described in Section 2. In the remainder of this section, we provide an overview of installation and dataset loading, as well as details of the implemented algorithms and methods contained in the library components shown in Fig. 1.

\subsection{Installation and Software Language}

In order to create the open-source multi-platform tool described in this paper, RR-APET, the Python programming language was selected for implementation. However, as the program is packaged as a complete, stand-alone executable file, it is able to be executed without a Python IDE (Integrated Development Environment) installed on the computer. RR-APET is available for the following operating systems (OS):

1. Windows

2. Linux

3. Mac OS

RR-APET is available at https://gitlab.com/ MegMcC/rr-apet-hrv-analysis-software as a unique compressed folder for each OS listed. Once downloaded, RR-APET can be operated by extracting the contents of the compressed folder to an appropriate location, then launching the executable program file titled 'RR-APET'. This folder also contains a copy of RR-APET's user guide manual, which contains detailed information on every aspect of RR-APET and it's functions.

\subsection{Loading the dataset and input data formats}

In the context of this work, a dataset is considered to be a collection of ECG recordings, stored in a usable format, and is the data input to the software for processing. To make the program as useful as possible, datasets can be imported in a number of formats. The following file formats are supported:

1. ASCII data files

2. Matlab MAT files

3. HDF5 files

4. WFDB files

The data set can be in row or column format, with each constituting a vector of ECG signal values, and can optionally include a time vector. By default the RR-APET software will select the first non-time vector for its initial display, however the user is able to switch between vectors or leads as desired using the provided Input drop-down menu. In this way, a user is able to load, view and annotate a full 12 lead ECG if desired.

When the time vector is included within the ECG data, it is automatically detected by the program and the user is not required to enter a sampling frequency. However, if the user chooses to input data that does not include a time vector, the user will be prompted to enter the sampling frequency ${ }^{5}$ manually, as it is not detectable from the supplied data. Error detection is included in this stage, for example, if the user mistakenly enters a sampling frequency which equates to the detection unlikely heartrates (such as $\mathrm{HR}<40 \mathrm{bpm}$ or $\mathrm{HR}>200 \mathrm{bpm}$ ), a warning will appear asking the user if they are happy to continue with the analysis of the imported data.

Additionally, annotations identifying the locations of Rpeaks within each ECG signal, can be loaded from a file. These series can also be stored in any of the above formats,

\footnotetext{
${ }^{5}$ Here we measure sampling frequency in Hertz, which equates to the number of samples per second
} 


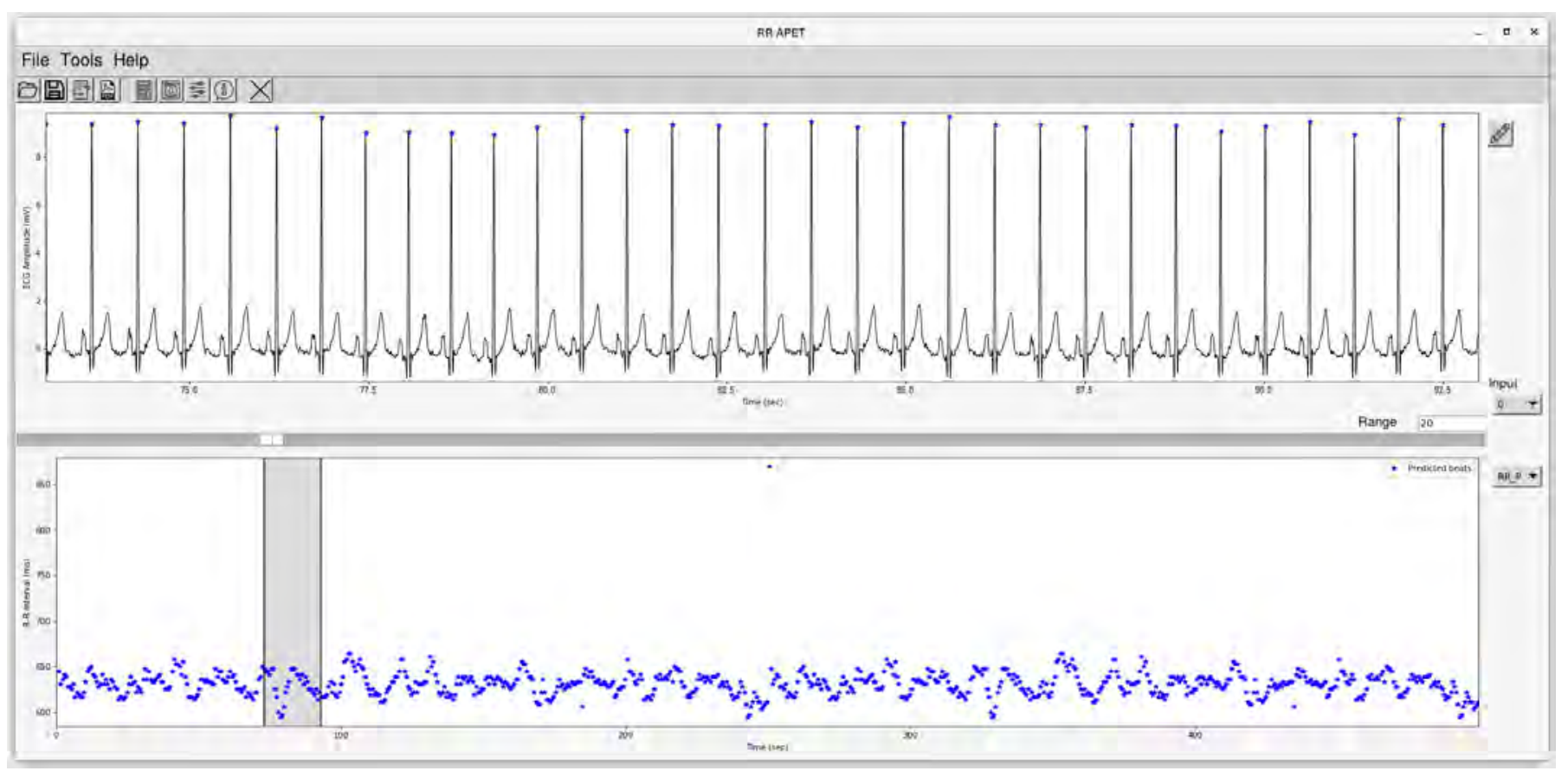

Fig. 5: The main operating window of RR-APET's graphical user interface.

and should be represented as a list of sample numbers or time values corresponding to the R-peaks for each ECG record in the data set, or as a list of the $\mathrm{RR}$ intervals. Furthermore, this file can be the same file that stores the data set, or a separate file altogether. The annotations can be both time values or vector sample numbers.

\subsection{The user interface}

RR-APET is fully operated through its graphical user interface (GUI) which can be divided into three key parts: (1) Data platform, (2) Algorithm and HRV metrics viewer, and (3) Plot Viewer. The data platform forms the main GUI window as shown in Fig. 5, and the Algorithm and HRV metrics viewer and Plot Viewer open as secondary pop-up windows when activated, as shown in Fig. 6 and Fig. 7, respectively. The functionalities incorporated into each of these sections are described below.

\subsubsection{Data platform}

The data platform is comprised of two key sections: the data browser, and the functionalities specific to controlling the data browser. The data browser can be further broken down into another two key sections: the ECG plot (top) and the RRI plot (bottom). The data displayed here shows the raw ECG signal, as selected by the user, and it's corresponding RRI series, as predicted by RR-APET's software. In order for the ECG data to be able to be interpreted by the user the time range is limited along the x-axis. The RRI series segment that correlates to data range shown in the current viewing window of the ECG axis, is indicated by the grey section on RRI plot.
The viewable ECG data can be controlled along the $\mathrm{x}$ axis using the slider function, or by adjusting the range with the entry box labelled 'Range (s)'. The user can also left click on an area of interest within the RRI series, and the ECG plot will be updated to be centered around the desired location. This is a particularly useful function, as a user can easily click on any outlier values present within the RRI series to identify the cause of the disturbance (e.g. R-peak detection error or abnormal heart function).

The functionalities specific to controlling the data browser, which are located on the right hand side of RR-APET's GUI for ease of use purposes, include: Edit Mode, the Input selection drop-down menu, and the Annotation selection drop-down menu. The Edit Mode function enables the user to correct misdetected R-peak locations, and thereby correct the correlated RRI values. This function is activated by left-clicking the pencil icon ( ). While activated, the user can add or remove points within the provided or predicted annotations for a given signal manually, with the resulting annotations able to be saved for later use. The Input selection drop-down menu enables the user to switch between channels when the input ECG file contains more that one data vector. This is particularly useful when analysing a classic 12 lead ECG. Finally, the Annotation selection drop-down menu allows the user to control which set of annotations are displayed on the RRI plot. The three options include; Predicted R-peak intervals (RR_P), Pre-annotated loaded R-peak intervals (RR_A), or both (RR_B). Here the default setting is $R R \_P$, and the options $R R \_A$ and $R R \_B$ only become available once the user has loaded an RRI series into the software. 


\begin{tabular}{|c|c|c|c|c|c|c|c|c|c|c|c|c|}
\hline \multicolumn{10}{|c|}{ Algorithm and HRY Metrics } & & & - \\
\hline \multicolumn{2}{|c|}{ Time-Domain Parameters } & \multicolumn{4}{|c|}{ Frequency-Domain Parameters } & \multicolumn{4}{|c|}{ Nonlinear Parameters } & \multicolumn{3}{|c|}{ Detection Algorithm Metrics } \\
\hline SDNN (m5) & 44.37 & & Welch & $\checkmark$ & & Recurrence & Analysis & Poincare & Analysis & Sensitivity $(\%)$ & 100.00 & \\
\hline SDANN (ms) & 40.66 & Peak Frequency & $\mathrm{VLF}(\mathrm{Hz})$ & $\mathrm{LF}(\mathrm{Hz})$ & $\mathrm{HF}(\mathrm{Hz})$ & $\operatorname{REC}(\%)$ & 34.93 & SD1 (ms) & 33.691 & Posilive Predictabifity (\%) & 100.00 & \\
\hline Mean RR interval (ms) & 850.25 & Peak Frequency & 0.000 & 0.040 & 0.166 & DET $(\%)$ & 96.53 & $\mathrm{SD} 2$ (ms) & 52.919 & Accuracy $(\%)$ & 100.00 & \\
\hline RMSSD (mis) & 47.62 & Percentage Power & VLF $(\%)$ & LF (\%) & $\begin{array}{l}0.166 \\
H F(\%)\end{array}$ & LAM (\%) & 92.23 & & & Detection Error Rate (\%) & 0.00 & \\
\hline pNN50 (96) & 17.90 & Percentage Power & 36.82 & 15.95 & 47.24 & Lmean (bts) & 10.04 & DFA & & Precision Window (ms) & 150.00 & Update \\
\hline & & Absolute Power & $V L F\left(m s^{\circ} 2\right)$ & LF $\left(m s^{\circ} 2\right)$ & $\mathrm{HF}(\mathrm{ms} \sim 2)$ & Lmax (bts) & 337 & $\alpha 1$ & 0.77 & & & \\
\hline & & & 681.52 & 295.18 & 874.48 & Vmean (bts) & 4.22 & & & & & \\
\hline & & LF/HF Ratio & 0.338 & & & & & & & & & \\
\hline & & & & & & & & & & & & Save \\
\hline
\end{tabular}

Fig. 6: The results viewer window from the graphical user interface of RR-APET; generated as described in Section 4.

Alternatively, the user can also choose to load data from devices such as mobiles, smart watches and strap sensors that do not output an ECG signal, but instead record an accurate $\mathrm{HR}$ signal generated by accurately detecting the interbeat intervals. To implement this feature, the user must manually change the import data type setting, located within the program preferences, from ECG to RRI. When this option is selected, the user will see just the RRI plot in the data browser, as the ECG data is unavailable. The Edit Mode function is still available to remove outlier peak values, however the input and annotation selection drop-down menus are removed to avoid confusion, as they are no longer applicable.

In addition to data visualisation, the data platform can be manipulated to perform a variety of functions using the operational control panel. The operation control panel is located in the upper left hand corner of the main GUI for RR-APET. It is formed by the combination of a menu bar and a quick-select toolbar, as seen at the top of Fig. 5. This panel includes a variety of functional controls common to many software packages, such as buttons that load the data, save the output, open the program preferences, open the help file and close the current window.

The program preferences option can be used to accomplish a variety of tasks, which include: updating the size and font of the displayed text, selecting and updating the settings for the R-peak detection algorithm implemented by RR-APET, updating the settings for the HRV analysis algorithms, and customising the import data settings. This feature also allows users implement and trial their own detection algorithm. Any R-peak detection algorithm of interest can be implemented in RR-APET's GUI by modifying the 'Template_function.py' python file provided and selecting 'Own Method' as the R-peak detection algorithm. For further detail on the program preferences, as well as all other previously listed options, is provided in RR-APET's user manual.

\subsubsection{Results viewer}

The results viewer operates as two additional pop-up windows that are generated when the user selects the 'Calculate Metrics' option from the operational control section. The first is the Algorithm and HRV Metrics window, shown in Fig. 6. The results displayed here are

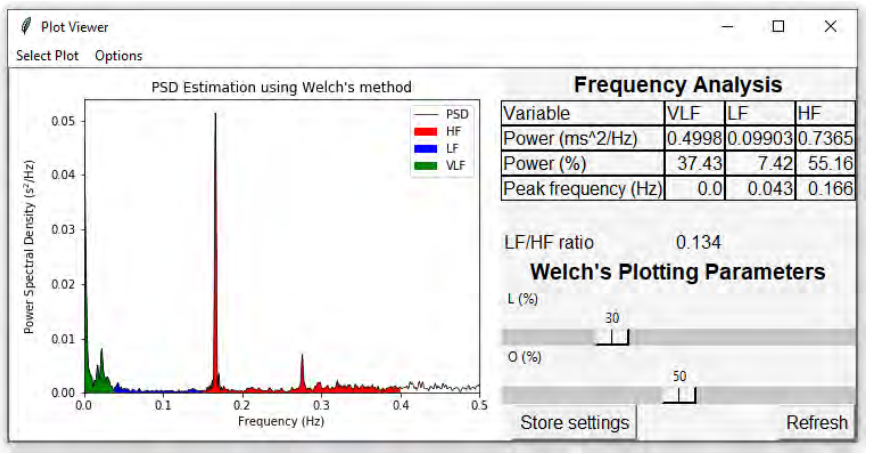

Fig. 7: The plot viewer window from the graphical user interface of RR-APET.

divided into four key sections: time-domain parameters, frequency-domain parameters, nonlinear parameters, and detection algorithm metrics. Within each section the results are displayed in a tabular format with units where appropriate. The frequency-domain results are calculated using Welch's periodogram by default, but can be updated by selecting the desired method by using the drop-down menu provided. Similarly, the detection algorithm metrics are calculated with a default precision window of $15 \mathrm{~ms}^{6}$, but this can be updated using the entry box located within this section. These metrics can be saved using ASCII text, Matlab MAT, or Python HDF5 file format.

The second pop-up is the Plot Viewer window shown in Fig. 7. This window can be used to view any of the graphical displays described in Section 2.2, including: the Welch, Blackman-Tukey and Lomb-Scargle periodogram, the AR spectrum, the Poincaré plot, the detrended fluctuation analysis plot, and the recurrence analysis plot. The figure of interest can be selected using the select plot dropdown menu. Displayed to the right hand side of the plot are the domain-relevant parameters, as well as the variable factors used to generate the plot. The variable parameters can be altered using the sliders or entry boxes provided on the lower right hand side of the window to determine the effect they have on the generated image. Additionally,

\footnotetext{
${ }^{6}$ Although entered in ms, the precision window also relies upon sampling frequency and therefore will round the the number of samples closest to the desired time.
} 
these images can be saved individually in multiple formats which include: encapsulated post script (.eps), portable network graphic (.png), or scalable vector graphic (.svg). The results viewer windows can be exited and relaunched at any time during the analysis or editing of annotations.

\section{Sample run}

\subsection{Single ECG test}

As a sample run, HRV analysis was conducted for a patient selected at random from the MIT-BIHA database $[21,42]$. The sample ECG, taken from patient \#111, is a 30 minute signal originally measured from a middle-aged female with first degree atrioventricular block. As this sample came from an annotated database, we were then able to load the original annotations, thereby allowing us to concurrently examine the effectiveness of the default detection algorithm. Alternatively, we could have chosen to edit the RRI series using the edit function to ensure the artefacts created by the misidentification of R-peaks were removed. From here we generated our HRV parameters using the default values set in the program.
The quantified time-domain, frequency-domain and nonlinear analysis parameters could then be viewed in the Algorithm and HRV Metrics window of the GUI as shown in Fig. 6. These metrics were saved by selecting the 'Save' button located in the bottom right hand corner of the Algorithm and HRV Metrics window, and using the file menu to enter a file name, and select both the appropriate location and image type (example given is type *.txt from the listed options). When saved using the text file format, the results are presented in column format, as seen in Fig. 8. The desired figures were then saved by simply selecting the 'Save' button located within the Options segment of the menu-bar, then using the file menu to enter a file name, and select both the appropriate location and image type (we chose *.eps from the listed options).

\subsection{Batch-processing test}

RR-APET is also able to be operated to examine the HRV metrics of multiple ECGs in a single process, which is also known as batch processing. We will now detail the steps required to perform multiple ECG analysis. First,

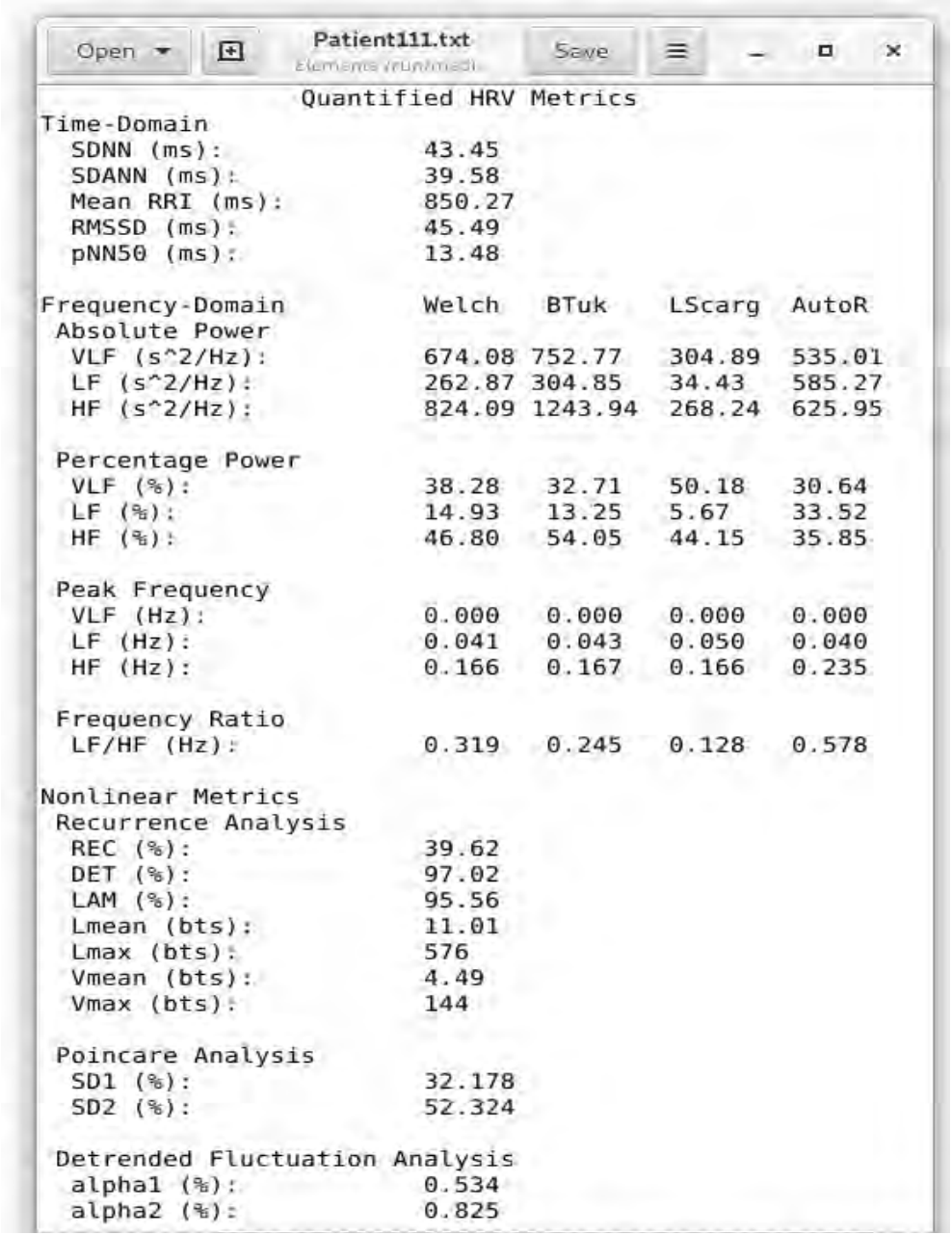

Fig. 8: Quantified HRV metrics saved in the default text file format. 


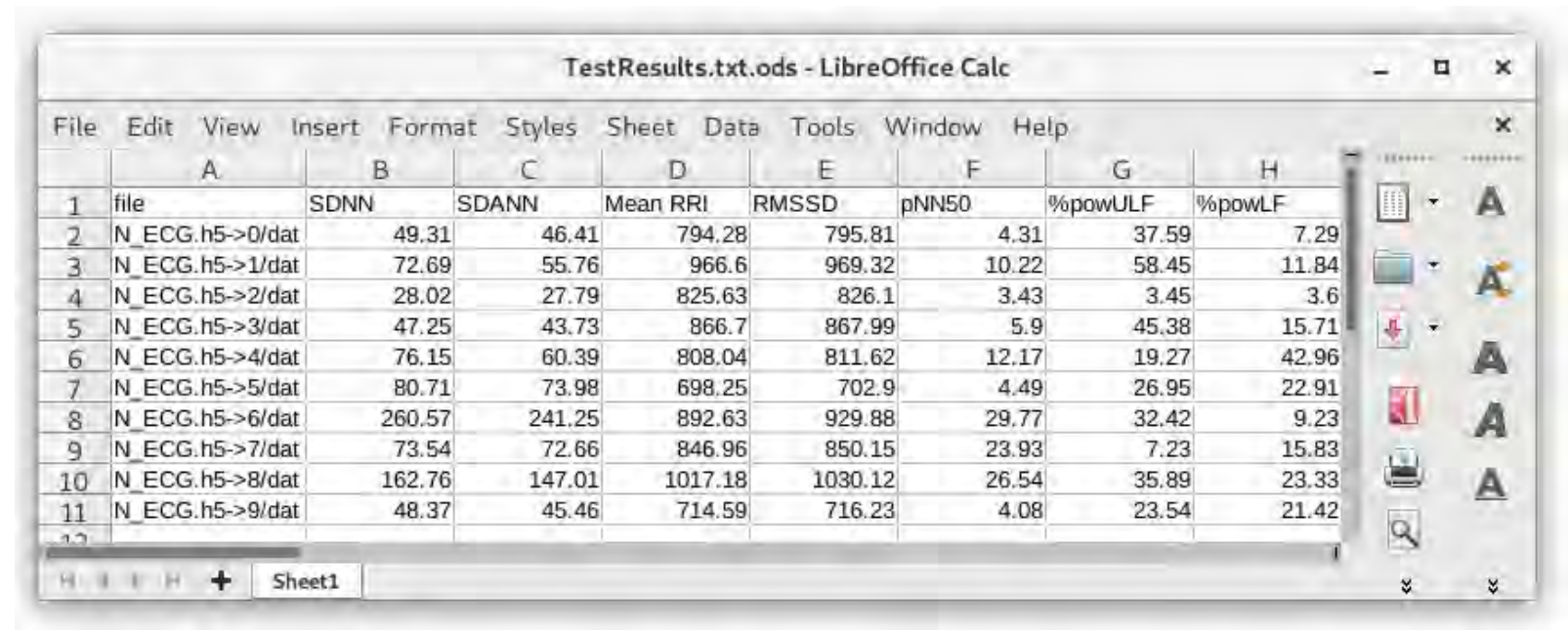

Fig. 9: Example output produced using the batch save HRV metrics option.

\begin{tabular}{|c|c|c|}
\hline \multicolumn{2}{|c|}{ Select File Type and Sampling Rate } & $\square$ \\
\hline Input Data Type & Output File Specifications & Sampling Rate (Fs) \\
\hline $\begin{array}{l}\text { Select the format of the input } \\
\text { ECG data: }\end{array}$ & $\begin{array}{l}\text { Enter the name of the output file } \\
\text { (with file type): }\end{array}$ & $\begin{array}{l}\text { Enter the sampling rate of the } \\
\text { ECG database selected: }\end{array}$ \\
\hline Text files *t.tt & 1 & $\mid$ \\
\hline & & Cancel \\
\hline
\end{tabular}

Fig. 10: Example of the pop-up window generated when using RRAPET's batch processing functionality.

save all ECG signals ${ }^{7}$ of interest into a single directory, making sure to use an accepted format (*.txt, *.mat, *.wfdb, or *.h5). Next, activate the multi-run popup window (shown in Fig. 10), by selecting the 'batch save' option from the quick-select toolbar or Tools Menu. Using the multi-run pop window, specify the file type of interest for the input ECG data. Then specify the output file name and type in the Output File Specifications entry box. Finally, specify the sampling frequency in the Sampling Frequency (Fs) and press 'Ok'. The metrics will be calculated using the detection algorithm and analysis parameters set within the program preferences, and stored according to the output file type provided. An example of a text file output produced using the batch processing functionality of RR-APET ${ }^{8}$ is shown in Fig. 9.

\section{Results}

\subsection{Evaluation of $R$-peak Detection Algorithms within $R R$-APET software}

The performance of each detection algorithm provided in the RR-APET software package was evaluated against the performance of the popular software package $\mathrm{Ku}$ bios [12], using the MIT-BIHA database [21, 42]. The

\footnotetext{
${ }^{7}$ Ensure all signals are recorded at the same sampling frequency.

${ }^{8}$ This text file has been viewed using LibreOffice Calc (the Linux equivalent to Microsoft's Excel)
}

performance of each method was rated using detection algorithm analysis metrics: Se, P+, DER, and Acc (originally described in Section 2.2.1). These metrics were calculated using the WFDB bxb comparator with the standard settings, and averaged across the database to produce the metrics shown in Table 1. It should be noted that no detection algorithm parameters were changed between patients, and that the performance of Kubios was calculated using it's default settings to avoid overfitting. Furthermore, the preset parameters used in RRAPET's detection algorithms were determined by testing the algorithms using various sources of data, including ECG signals sampled at various frequencies, before being evaluated against the standard MIT-BIHA database in order to ensure robustness across databases.

When analysing the data presented in Table 1, it is clear that the K-means clustering (KMCD) derived detection algorithm was competitive with the Kubios HRV software only in the $\mathrm{P}+$ metric and not the Se metric. Upon further investigation it was found that the KMCD method is not sensitive to peak polarity at all, meaning that it is unable to detect negatively orientated R-peaks and therefore is unreliable for ECGs which contain both positive and negative polarity R-peaks. However, the Pan-

Table 1: Performance comparison of proposed detection algorithms against established Kubios software using the MIT-BIHA database

\begin{tabular}{lcccc}
\hline Algorithm & $\begin{array}{c}\text { Se } \\
(\%)\end{array}$ & $\begin{array}{c}\text { P+ } \\
(\%)\end{array}$ & $\begin{array}{c}\text { DER } \\
(\%)\end{array}$ & $\begin{array}{c}\text { Acc } \\
(\%)\end{array}$ \\
\hline Pan-Tompkins $^{\mathrm{a}}$ & 99.10 & 99.52 & 1.62 & 98.63 \\
$\begin{array}{l}\text { K-Means } \\
\text { Clustering }\end{array}$ & 94.45 & 99.12 & 19.16 & 93.68 \\
Hilbert Transform $^{\mathrm{a}}$ & 99.42 & 99.27 & 0.80 & 99.26 \\
Kubios $^{\mathrm{b}}$ & 98.09 & 99.82 & 2.49 & 97.92 \\
\hline a Our implementation within RR-APET & derived \\
$\quad \begin{array}{l}\text { from original works listed } \\
\text { b Implemented with default settings selected. }\end{array}$
\end{tabular}


Tompkins derived (PTD) and Hilbert transform derived (HTD) algorithms were both highly competitive with the Kubios HRV software in all metrics. Upon further investigation into the PTD method, it was found that this method is quite effective at identifying QRS-complexes, but not as effective at identifying fiducial marker (i.e R-peak). In comparison, the HTD method was able to more consistently identify the fiducial marker within the QRS complexes, and was therefore selected as the default algorithm to be implemented within the RR-APET software package.

Table 2: Performance comparison of HRV metrics generated within $R R-A P E T$ (RRA) against the same metrics generated by established Kubios (Kub) software using randomly selected patients from the MIT-BIHA database.

\begin{tabular}{|c|c|c|c|c|c|c|}
\hline \multirow[b]{2}{*}{ Metrics } & \multicolumn{2}{|c|}{ \#111 } & \multicolumn{2}{|c|}{$\# 123$} & \multicolumn{2}{|c|}{$\# 221$} \\
\hline & RRA & Kub & RRA & Kub & RRA & Kub \\
\hline SDNN & 43.45 & 43.46 & 115.28 & 115.33 & 262.72 & 262.81 \\
\hline SDANN & 39.58 & NA & 113.68 & NA & 259.10 & NA \\
\hline$\overline{\mathrm{RRI}}$ & 850.3 & 850.3 & 1191.8 & 1191.9 & 826.3 & 826.2 \\
\hline RMSSD & 45.49 & 45.51 & 102.72 & 102.72 & 345.91 & 346.34 \\
\hline pNN50 & 13.52 & 13.53 & 63.41 & 63.32 & 81.32 & 81.32 \\
\hline $\mathrm{VLF}_{a b s}$ & 674 & 622 & 3401 & 3652 & 18600 & 18864 \\
\hline $\mathrm{LF}_{a b s}$ & 263 & 351 & 5169 & 6290 & 17300 & 15763 \\
\hline $\mathrm{HF}_{a b s}$ & 825 & 1010 & 2402 & 2614 & 35470 & 31648 \\
\hline $\mathrm{VLF}_{\%}$ & 38.26 & 31.35 & 31.00 & 29.08 & 26.06 & 28.40 \\
\hline $\mathrm{LF}_{\%}$ & 14.94 & 17.67 & 47.11 & 50.09 & 24.24 & 23.73 \\
\hline $\mathrm{HF}_{\%}$ & 46.80 & 50.90 & 21.89 & 20.82 & 49.70 & 47.65 \\
\hline $\mathrm{VLF}_{\text {fpeak }}$ & 0.000 & 0.003 & 0.037 & 0.037 & 0.000 & 0.003 \\
\hline $\mathrm{LF}_{\text {fpeak }}$ & 0.041 & 0.040 & 0.047 & 0.050 & 0.122 & 0.123 \\
\hline $\mathrm{HF}_{\text {fpeak }}$ & 0.166 & 0.167 & 0.273 & 0.273 & 0.298 & 0.383 \\
\hline $\mathrm{LF} / \mathrm{HF}$ & 0.319 & 0.347 & 2.153 & 2.406 & 0.488 & 0.498 \\
\hline REC & 39.62 & 35.51 & 28.89 & 28.09 & 20.38 & 18.91 \\
\hline DET & 97.02 & 97.20 & 96.45 & 97.21 & 93.41 & 94.91 \\
\hline LAM & 95.56 & NA & 88.90 & NA & 70.71 & NA \\
\hline $\mathrm{L}_{\text {mean }}$ & 11.01 & 11.22 & 9.55 & 9.00 & 7.81 & 7.29 \\
\hline $\mathrm{L}_{\max }$ & 576 & 400 & 141 & 139 & 126 & 75 \\
\hline $\mathrm{V}_{\text {mean }}$ & 4.49 & NA & 4.92 & NA & 5.31 & NA \\
\hline $\mathrm{V}_{\max }$ & 144 & NA & 144 & NA & 146 & NA \\
\hline $\mathrm{SD}_{1}$ & 32.18 & 32.19 & 72.68 & 72.66 & 244.71 & 244.65 \\
\hline $\mathrm{SD}_{2}$ & 52.32 & 52.35 & 145.7 & 145.9 & 279.68 & 279.62 \\
\hline$\alpha_{1}$ & 0.603 & 0.797 & 1.17 & 1.18 & 0.581 & 0.616 \\
\hline$\alpha_{2}$ & 0.787 & 0.713 & 0.629 & 0.600 & 0.711 & 0.738 \\
\hline
\end{tabular}

* All frequency-domain metrics were calculated using the Welch periodogram method.

\subsection{Validation of analytic HRV metrics}

The validity of the analytic HRV metrics generated within RR-APET's software package was established by evaluation against the known and trusted HRV software package Kubios [12]. Three patients were selected at random from the MIT-BIHA database for comparison, to avoid loss of information through average performance metrics. As seen in Table 2, the time-domain parameters were highly similar across both platforms with the greatest difference occurring in the $R M S S D$ parameter for patient \#221, where the difference was less than $0.5 \mathrm{~ms}$. The frequency-domain parameters, while similar, were not identical between platforms. This is likely due to the different pre-processing parameters used when preparing the RRI series for Fourier analysis; which is required for frequency analysis using Welch's method as the RRI series is not an evenly sampled series. However, both platforms still indicate similar trends in the frequency domain, with a maximal difference of $7 \%$ occurring for patient \#111 in the VLF band. Finally, the nonlinear parameters produced using both platforms were also very similar.

\subsection{User evaluation of RR-APET}

In order to evaluate the users' assessment of the RRAPET application, we conducted a usability questionnaire. Participants included several volunteers with either a medical or engineering based background of various ages and ethnicities. These participants were asked to complete a series of challenges before rating the software. The questionnaire was presented using a series of common software usability questions and a Likert Scale response, where $1=$ Totally disagree and $5=$ Totally agree. The results of this questionnaire are shown in Table 3 .

Table 3: RR-APET Usability Questionnaire.

\begin{tabular}{lc}
\hline Statement & Rating \\
\hline It is simple to use & 3.71 \\
It is user friendly & 4.00 \\
It requires the fewest steps possible to & 4.57 \\
accomplish what I want to do with it & \\
It is flexible & 3.86 \\
Using it is effortless & 3.29 \\
\hline I can use it without the written instructions & 4.29 \\
I don't notice any inconsistencies as I use it & 4.14 \\
I can recover from mistakes quickly and easily & 4.43 \\
I learned to use it quickly & 4.43 \\
I easily remember how to use it & 4.57 \\
It is easy to learn to use it & 4.43 \\
I quickly became skillful with it & 4.14 \\
\hline Average Rating & $\mathbf{4 . 1 6}$ \\
\hline
\end{tabular}




\section{Discussion}

In this paper, RR-APET's user-friendly and opensource HRV analysis software was introduced. This versatile software can be used for a variety of functions, ranging from the analysis of R-peak detection algorithms, to the analysis of the RRI signal these algorithms produce. Unlike other HRV analysis tools that often restrict the user to a single detection algorithm, RR-APET contains a choice of several established detection algorithms, as well as a template function built into the GUI, which can be easily edited to test any new algorithms as desired. The performance of these algorithms can be visually discerned using the GUI, or quantified using the standard sensitivity, positive predictability, accuracy, and detection error rate metrics, where true annotations are provided. Additionally, the easy-to-use editing function can be implemented alongside the prediction algorithms to quickly and effectively produce both accurate and precise annotation files for any given ECG signal.

RR-APET also includes a wide variety of HRV metrics that can be used to evaluate RRI signals, including metrics from the time and frequency-domains, and nonlinear metrics. These metrics are computed according to well established standards in HRV literature [2, 5, 27], and therefore are comparable to metrics produced in other HRV studies. Notably, while this software is available as an executable GUI, the python scripts used to create this platform as also open source. This means that inexperienced users can operate the software without the need to alter any scripts, but experienced coders are able to access the build files to modify the program at any point in order to better suit their specific needs. Furthermore, the user is able to select their preferred algorithm from the detection algorithms provided within, which can also updated using the program preferences if desired. This algorithm can then used to analyse multiple ECG files without any further user interaction. This is particularly beneficial for those who want to use large HRV metric datasets for secondary studies, i.e. the correlation between HRV and any number of illnesses. It should also be noted that, while this is the first version of RR-APET's software, it will continue to be updated and improved to contain more features and analysis options based upon user feedback and suggestions. Furthermore, RR-APET as is an open-sourced, Python-based platform, any user can simply download and tweak the software at their discretion.

Emphasized here however, are a few points which can have a significant effect on the analysis results yielded from the software platform. Firstly, the performance of the R-peak detection algorithms provided within, like all others presented in current literature, are dependent on the signal quality of the data provided. R-peak detection algorithms are often most susceptible to reduced performance (i.e. increased artefacts) when arrhythmia's are present, however, other sources of noise such as electrode motion or AC coupling can be equally detrimental to overall performance. Often, detection algorithms that are robust to certain types of noise, are more susceptible to others, and so care should be taken to ensure that the detection algorithm of choice is performing as expected before HRV analysis is performed. Secondly, whilst the in-depth graphical analysis component of the software is provided in the GUI, it is not included by default in the batch processing option, due to the additional time and space constraints required; however, can be easily added back in at the users discretion.

\section{Conclusion}

RR-APET is a useful, open-source, Python-based GUI tool that can be used for the analysis of both R-peak detection algorithms and HRV. By providing a variety of quantified metrics and visual aids, this software platform offers a robust base for further research to be conducted, particularly in the field of correlating patient healthcare outcomes to HRV parameters. New versions of this software will continue to be developed in order to improve its performance, and offer new features.

\section{Acknowledgment}

The authors would like to thank the anonymous reviewers whose comments have greatly improved this manuscript.

\section{References}

[1] D. Murray, "What is heart rate variability and is it blunted by tumor necrosis factor?," Chest Journal, vol. 123, pp. 664-667, March 2003.

[2] T. F. of The European Society of Cardiology, T. N. A. S. of Pacing, and Electrophysiology, "Heart rate variability standards of measurement, physiological interpretation, and clinical use," European Heart Journal, vol. 17, pp. 354-381, 1996.

[3] R. E. Kleiger, J. P. Miller, J. T. Bigger Jr., and A. J. Moss, "Decreased heart rate variability and its association with increased mortality after acute myocardial infarction," The American Journal of Caridiology, vol. 59, pp. 256-262, February 1987.

[4] M. T. La Rovere, J. T. Bigger Jr., F. I. Marcus, A. Mortara, and P. J. Schwartz, "Baroreflex sensitivity and heart-rate variability in prediction of total cardiac mortality after myocardial infarction," The Lancet, vol. 351, pp. 478-484, February 1998.

[5] P. Ghasemi and M. R. Raoufy, "Prediction of mortality in patients with sepsis using detrended fluctuation analysis of heart rate variability," in 2016 23rd Iranian Conference on Biomedical Engineering and 2016 1st International Iranian Conference on Biomedical Engineering (ICBME), pp. 150-154, IEEE, 2016.

[6] J. S. Perkiömäki and H. V. Huikuri, "Applying heart rate variability in clinical practice following acute myocardial infarction," in ECG Time Series Variability Analysis, pp. 389402, CRC Press, 2017.

[7] R. L. Conder and A. A. Conder, "Heart rate variability interventions for concussion and rehabilitation," Frontiers in psychology, vol. 5, p. 890, 2014. 
[8] T. P. Beauchaine and J. F. Thayer, "Heart rate variability as a transdiagnostic biomarker of psychopathology," International Journal of Psychophysiology, vol. 98, no. 2, pp. 338-350, 2015.

[9] J.-G. Dong, "The role of heart rate variability in sports physiology," Experimental and therapeutic medicine, vol. 11, no. 5, pp. 1531-1536, 2016.

[10] F. Shaffer and J. P. Ginsberg, "An overview of heart rate variability metrics and norms," Frontiers in public health, vol. 5, no. 258, 2017.

[11] K. Oy, "Kubios," 2016-2019.

[12] M. Tarvainen, J. Niskanena, J. Lipponena, P. Ranta-ahoa, and P. Karjalainena, "Kubios HRV - Heart rate variability analysis software," Computer Methods and Programs in Biomedicine, vol. 113 , no. 1 , pp. $210-220,2014$.

[13] N. Instruments, "What is labview?," 2019.

[14] P. W. Biosignals, "We create innovative products, for oems, industry \& researchers," 2019.

[15] L. Mourot, "Codesna_hrv, a new tool to assess the activity of the autonomic nervous system from heart rate variability," Physical Medicine and Rehabilitation Research, vol. 3, no. 1, pp. 2-6, 2018.

[16] MATLAB, version R2018a. Natick, Massachusetts: The MathWorks Inc., 2018.

[17] J. Vicente, L. Johannesen, L. Galeotti, and D. G. Strauss, "Ecglab: User friendly ecg/vcg analysis tool for research environments," in Computing in Cardiology 2013, pp. 775-778, IEEE, 2013.

[18] P. Perakakis, M. Joffily, M. Taylor, P. Guerra, and J. Vila, "Kardia: A matlab software for the analysis of cardiac interbeat intervals," Computer methods and programs in biomedicine, vol. 98, no. 1, pp. 83-89, 2010.

[19] T. Kaufmann, S. Stterlin, S. Schulz, and C. Vgele, "Artiifact: a tool for heart rate artifact processing and heart rate variability analysis," Behaviour Research Methods, vol. 43, no. 4, pp. 1161$1170,2011$.

[20] M. Vollmer, "Hrvtool - analyzing heart rate variability," 20152019.

[21] A. Goldberger, L. Amaral, L. Glass, J. Hausdorff, P. Ivanov, R. Mark, J. Mietus, G. Moody, C.-K. Peng, and H. Stanley, "Physiobank, physiotoolkit, and physionet," Circulation, vol. 101, pp. 215-220, June 2000.

[22] L. Rodríguez-Liñares, A. J. Méndez, M. J. Lado, D. N. Olivieri, X. Vila, and I. Gómez-Conde, "An open source tool for heart rate variability spectral analysis," Computer methods and programs in biomedicine, vol. 103, no. 1, pp. 39-50, 2011.

[23] L. Rodríguez-Liñares, M. J. Lado, V. X.A., A. Méndez, and C. P, "ghrv: Heart rate variability analysis made easy," Computer methods and programs in Biomedicine, vol. 116, no. 1, pp. 26-38, 2014.

[24] J. Pan and W. Tompkins, "A real-time QRS detection algorithm," IEEE transactions on biomedical engineering, vol. BME-32, pp. 230-236, March 1985.

[25] M. Merino, I. Gomez, and A. Molina, "Envelopment filter and K-means for the detection of QRS waveforms in electrocardiogram," Medical Engineering and Physics, vol. 37, no. 6, pp. 605-609, 2015.

[26] S. Thulasi Prasad and S. Varadarajan, "Heart rate detection using Hilbert transform," IJRET: Int. J. of Research in Engeering and Technology, vol. 2, no. 8, pp. 508-513, 2013.

[27] J. P. Zbilut, N. Thomasson, and C. L. Webber, "Recurrence quantification analysis as a tool for nonlinear exploration of nonstationary cardiac signals," Medical Engineering $\&$ Physics, vol. 24, pp. 53-60, 2002.

[28] J. Saul, P. Albrecht, R. Berger, and R. Cohen, "Analysis of long term heart rate variability: methods, $1 / \mathrm{f}$ scaling and implications," Computers in Cardiology, vol. 14, pp. 419-422, 1988.

[29] P. Welch, "The use of fast fourier transform for the estimation of power spectra: a method based on time averaging over short, modified periodograms," IEEE Transactions on audio and electroacoustics, vol. 15, no. 2, pp. 70-73, 1967.
[30] S. Haykin, Nonlinear Methods of Spectral Analysis. Topics in Applied Physics, Springer Science \& Business Media, 2006.

[31] J. Makhoul, "Linear predicition: A tutorial review," Proceedings of the IEEE, vol. 63, no. 4, pp. 561-580, 1975.

[32] T. Ruf, "The lomb-scargle periodogram in biological rhythm research: analysis of incomplete and unequally spaced timeseries," Biological Rhythm Research, vol. 30, no. 2, pp. 178-201, 1999.

[33] G. E. Billman, "The lf/hf ratio does not accurately measure cardiac sympatho-vagal balance," Frontiers in physiology, vol. 4, Feb 2013.

[34] M. de Godoy, "Nonlinear analysis of heart rate variability: A comprehensive review," Journal of Cardiology and Therapy, vol. 3, no. 3, pp. 528-533, 2016.

[35] J. Eckmann, S. Kamphorst, and D. Ruelle, "Recurrence plots of dynamical systems," Europhysics Letters, vol. 4, pp. 973-977, November 1987.

[36] C. Webber and J. Zbilut, "Dynamical assessment of physiological systems and states using recurrence plot strategies," Journal of Applied Physiology, vol. 76, no. 2, pp. 965-973, 1994.

[37] N. Marwan, N. Wessel, U. Meyerfeldt, A. Schirdewan, and J. Kurths, "Recurrence-plot-based measures of complexity and their application to heart-rate-variability data," Physical Review E, vol. 66, p. 26702, August 2002.

[38] V. V. Morariu, L. Buimaga-Iarinca, C. Vamoş, and Ş. M. Şoltuz, "Detrended fluctuation analysis of autoregressive processes," Fluctuation and Noise Letters, vol. 7, no. 3, pp. L249-L255, 2007.

[39] F. Beckers, B. Verheyden, and A. E. Aubert, "Aging and nonlinear heart rate control in a healthy population," American Journal of Physiology-Heart and Circulatory Physiology, vol. 290, pp. H2560-H2570, November 2006.

[40] A. Schumacher, "Linear and nonlinear approaches to the analysis of $\mathrm{rr}$ interval variability," Biological research for nursing, vol. 5, no. 3, pp. 211-221, 2004.

[41] H. V. Huikuri, T. Seppnen, M. J. Koistinen, K. J. Airaksinen, M. Ikheimo, A. Castellanos, and R. J. Myerburg, "Abnormalities in beat-to-beat dynamics of heart rate before the spontaneous onset of life-threatening ventricular tachyarrhythmias in patients with prior myocardial infarction," Circulation, vol. 93, no. 10, pp. 1836-1844, 1996.

[42] G. Moody and R. Mark, "The impact of the mit-bih arrhythmia database," IEEE Engineering in Medicine and Biology, vol. 20, no. 3 , pp. 45-50, 2001. 